\title{
List of contributing authors
}

María Teresa Aguado Molina

Animal Evolution and Biodiversity

Johann-Friedrich-Blumenbach Institute for Zoology and Anthropology

Georg-August-Universität Göttingen, Göttingen, Germany

aguadomolina@uni-goettingen.de

\section{Charatsee Aungtonya}

Phuket Marine Biological Center

PO Box 60 , Phuket 83000 , Thailand

charatsee@hotmail.com

\section{Torkild Bakken}

Norwegian University of Science and Technology

NTNU University Museum

N0-7491, Trondheim, Norway

torkild.bakken@ntnu.no

\section{Markus Böggemann}

Universität Vechta

Fakultät II - Biologie

Driverstraße 22, Vechta 49377, Germany

Markus.Boeggemann@uni-vechta.de

\section{Polina Borisova}

P.P. Shirshov Institute of Oceanology

Russian Academy of Sciences

Russia

salixhastata@ya.ru

\section{Nataliya Budaeva}

Natural History Collections

University Museum of Bergen

P.O. Box 7800, NO-5020 Bergen, Norway

nataliya.budaeva@uib.no

\section{María Capa}

Departament de Biologia

Universitat de les Illes Balears

Palma, Illes Balears, Spain

maria.capa@uib.es

\section{Danny Eibye-Jacobsen}

Zoological Museum

Natural History Museum of Denmark

Universitetsparken 15, Copenhagen Ø DK-2100, Denmark

dejacobsen@snm.ku.dk

\section{María Ana Fernández-Álamo}

Facultad de Ciencias, UNAM

Departamento de Biologia Comparada

Av. Universidad 3000

Circuito Exterior S/N, Delegación Coyoacán, C.P. 04510,

Ciudad Universitaria, D.F. México

mafa@ciencias.unam.mx
Christopher J. Glasby

Museum and Art Gallery Northern Territory,

PO Box 4646,

Darwin, NT 0801, Australia

Chris.Glasby@magnt.net.au

\section{Brett C. Gonzales}

Smithsonian Institution

National Museum of Natural History

Department of Invertebrate Zoology

P.O.Box 37021, Washington, DC 20013-7012, USA

gonzalezb@si.edu

\section{Stéphane Hourdez}

Dir. Adjoint Laboratoire d'Ecogéochimie des Environnements

Benthiques (LECOB)

UMR 8222 CNRS-Sorbonne Université

Observatoire Océanologique de Banyuls

Avenue Pierre Fabre, 66650 Banyuls-sur-mer, France

hourdez@obs-banyuls.fr

\author{
Paulo Lana \\ Center for marine Studies -UFPR \\ Av Beira Mar s/h \\ Pontal do Sul \\ Parana 83255-000, Brazil \\ lana@ufpr.br
}

\section{Wagner F. Magalhães}

University of Hawaii at Manoa

2538 McCarthy Mall, Edmondson Hall 216, Honolulu, HI 96826, USA

wagnerfm@hawaii.edu

Tatiana Menchini Steiner

Departamento de Biologia Animal

Instituto de Biologia

Universidade Estadual de Campinas

Campinas, SP 13083-862, Brazil

tatims@unicamp.br

Karen J. Osborn

Department of Invertebrate Zoology

Smithsonian National Museum of Natural History

10th and Constitution Ave NW

Washington, DC 20560, USA

osbornk@si.edu

\section{Eivind Oug}

Norwegian Institute for Water Research

Region South

Jon Lilletuns Vei 3, Grimstad, Norway NO-4879, Norway

eivind.oug@niva.no 


\section{Günter Purschke}

Zoologie und Entwicklungsbiologie

Fachbereich Biologie/Chemie

Universität Osnabrück

Osnabrück 49069, Germany

gpurschk@uni-osnabrueck.de

\section{Ascensão Ravara}

Departamento de Biologia

Campus de Santiago

Universidade de Aveiro

Aveiro 3810-193, Portugal

aravara@ua.pt

\section{Alexandra Elaine Rizzo}

Universidade do Estado do Rio de Janeiro

Instituto de Biologia

Depto. de Zoologia Laboratorio de Zoologia de Invertebrados (sala 516)

Rua Sao Francisco Xavier, 524, CEP 20550-900 Maracana,

Rio de Janeiro - RJ, Brasil

aerizzo@hotmail.com

\section{Sergio I. Salazar-Vallejo}

El Colegio de la Frontera Sur

CONACYT, Unidad Chetumal, México.

savs551216@hotmail.com

\section{Guillermo San Martín}

Departamento de Biologiá

Facultad de Ciencias

Universida Autónoma de Madrid

Canto Blanca, Madrid 28049, Spain

guillermo.sanmartin@uam.es

\section{Cinthya S.G. Santos}

Universidade Federal Fluminense

Niteroi Rio de Janeiro, Brazil

cinthyasantos@id.uff.br

\section{Charlotte Watson}

Research Associate, Marine Invertebrates

Museum and Art Gallery of the Northern Territory

GPO Box 4646, Darwin, NT 0801; 19 Conacher St, The Gardens,

Darwin, NT 0820, Australia

Charlotte.Watson@magnt.net.au

\section{Robin S. Wilson}

Museum Victoria

GPO Box 666, Melbourne, Victoria 3001, Australia

rwilson@museum.vic.gov.au

\section{Katrine Worsaae}

Marine Biological Section

Department of Biology

University of Copenhagen

Universitetsparken 4, Copenhagen 2100, Denmark

worsaae@bio.ku.dk 
